\title{
Desarrollo y validación de una escala de medida de la empatía intercultural
}

\author{
Development and validation of a scale for measuring intercultural empathy
}

\author{
González-González, Hugo; Álvarez-Castillo, José-Luis \& Fernández-Caminero, Gemma \\ Universidad de Córdoba
}

\begin{abstract}
Resumen
La investigación y la práctica relacionadas con el uso de estrategias empáticas en entornos educativos multiculturales se está revelando como una de las más eficaces vías para la lucha contra el sesgo intergrupal. El objeto del presente estudio es diseñar un instrumento de medición de la empatía intercultural en su componente afectivo, específico para la población española y hacia la población de origen marroquí, que permita diagnosticar este rasgo o capacidad antes de aplicar estrategias educativas en entornos multiculturales. Con este propósito se utilizaron dos muestras de estudiantes universitarios para realizar una validación cruzada y, mediante análisis factorial exploratorio (utilizando el software Factor) y confirmatorio (SPSS-AMOS), se obtuvo una estructura de 3 factores: preocupación empática, comprensión y afecto. Los índices de bondad de ajuste, así como la validez y fiabilidad revelada por los análisis multidimensionales, demuestran que el específicos en los que se tenga en consideración el perfil de las poblaciones objeto de estudio en ámbitos multiculturales.
\end{abstract}

Fecha de recepción 21 de Diciembre 2015

Fecha de aprobación 27 de Noviembre 2015

Fecha de publicación 30 de Noviembre 2015

\section{Palabras clave:}

Empatía; comunicación intercultural; validación; sesgo intergrupal

\begin{abstract}
The research and practice connected to the use of empathic strategies in multicultural educational settings is emerging as one of the most effective ways to combat intergroup bias. The aim of this study is to design an instrument to measure the specific intercultural empathy in its affective component for the Spanish population towards the population of Moroccan origin. Thus, this instrument makes possible the measurement of this feature or capability before implementing educational strategies in multicultural contexts. Therefore, two samples of University students were used to carry out a cross validity study through exploratory (Factor 9.20) and confirmatory factor analysis (SPSS-AMOS). Then, a 3-factor structure was obtained: empathic concern, understanding and affection. The goodness of fit indices, together with the validity and reliability revealed by multidimensional analysis prove that the model is optimal. Eventually, it is discussed the desirability of designing specific instruments that take into account the profile of the populations under this study
\end{abstract}

\section{Keywords:}

Empathy; intercultural communication; validity; intergroup bias

Reception Date

2014 December 21

Approval Date

2015 November 27

Publication Date:

2015 November 30 
Acerca de la Empatía, la literatura refleja una considerable diversidad en lo que se refiere a la delimitación de dicho constructo (Fernández-Pinto, López-Pérez \& Márquez, 2008; Gerdes, Segal \& Lietz, 2010). Esta ha sido definida por Hoffman (1975, 1981, 1982, 1983) y por Strayer y Eisenberg (1987) como la experiencia afectiva vicaria de los sentimientos de otra persona. Sin embargo, las investigaciones revisadas demuestran que se trata de un constructo que admite distintas miradas desde multitud de prismas que comprenden componentes que, aunque similares y en algunos casos complementarios, pueden resultar de interés para el estudio desde diferentes vertientes: psicológica, sociológica, intercultural, patológica, de género, religiosa, etc. (Albiero \& Matricardi, 2013; Barnett \& Mann, 2013; Fernández-Pinto et al., 2008; Gerdes et al., 2010; Hardy, Walker, Rackham \& Olsen, 2012; Harris \& Picchioni, 2013; Wang et al., 2003).

A pesar de esta variedad de enfoques, se ha alcanzado cierto consenso en lo concerniente a determinar algunos correlatos conductuales de la empatía. Así, en los últimos años se ha puesto de relieve su importancia en la disposición prosocial de las personas (Belacchi \& Farina, 2012; Butrus \& Witenberg, 2013; Hodges, Clark \& Myers, 2011; Shen, Carlo \& Knight, 2013; Welp \& Brown, 2014) y su rol en el conflicto (Barnett \& Mann, 2013; Sanmartín, Carbonell \& Banos, 2011; Zembylas, 2013). En un trabajo de revisión de los estudios sobre el tema, Eisenberg (2000) planteaba la relevancia de la empatía en el desarrollo moral, entendida como una respuesta emocional que procede de la comprensión del estado o situación de otra persona, con el efecto de llegar a experimentar sentimientos semejantes a los del otro. Así, la respuesta empática incluye la capacidad para comprender a la otra persona y ponerse en su lugar a partir de lo que se observa, de la información verbal o de información accesible desde la memoria (toma de perspectiva), abarcando asimismo la reacción afectiva de compartir su estado emocional, lo que puede producir tristeza, malestar o ansiedad. La empatía así entendida desempeñaría un papel central en la disposición prosocial de las personas (Eisenberg, 2000). Recientemente Li, Mai y Liu (2014) han revisado la investigación sobre empatía y otros campos sociales: emoción, teoría de la mente o "mentalizing” y juicios morales; pero en este caso desde una perspectiva centrada en el funcionamiento a nivel neurológico y de interconexiones entre las distintas áreas del cerebro. En este trabajo se evidencia cómo la experimentación con neuroimagen ha contribuido a demostrar que, efectivamente, las personas utilizamos los recuerdos y asociaciones de experiencias pasadas como cimientos para comprender las emociones y estados cognitivos de los demás.

Si a este correlato unimos el vínculo de la empatía con el prejuicio, con la exclusión social y con las actitudes intergrupales, explícitas e implícitas (Albiero \& Matricardi, 2013; Li, Mai \& Liu, 2014; Shih, Stotzer \& Gutierrez, 2013; Shi, Trahan, Wang \& Stotzer, 2009), se comprende aún mejor la argumentación que ha servido para defender la investigación y la práctica relacionadas con el uso de estrategias empáticas en entornos educativos multiculturales (Belacchi \& Farina, 2012; Numata, 2013), o el empleo de diferentes programas de educación intercultural con el fin de incrementar la empatía (Peek \& Park, 2013; Todd, Bodenhausen \& Galinsky, 2012). En definitiva, esta representa un elemento clave en el establecimiento de relaciones sociales afiliativas, y es por ende percibida como una necesidad educativa en los contextos interculturales que actualmente caracterizan nuestras aulas y sociedades.

En este sentido, dada la relevancia socioeducativa de la empatía, se echa de menos, sin embargo, la existencia de instrumentos sencillos de calidad, válidos y fiables, que faciliten su medición tanto en entornos de investigación como de práctica educativa intercultural. En relación con la detección de esta necesidad surge el presente estudio, que pretende realizar una aportación al diagnóstico y evaluación de la empatía, con 
el consiguiente efecto facilitador sobre la planificación de intervenciones educativas más fieles al enfoque intercultural en ámbitos de diversidad.

\section{Medición de la empatía}

La empatía general ha sido evaluada con distintos tipos de instrumentos. Así, Fernández-Pinto y sus colegas (2008), en su revisión, analizan la siguiente tipología de medidas: un grupo de cuestionarios adoptan una perspectiva integradora (IRI, EQ, TECA); otros se centran en la dimensión afectiva (QMEE, BEES, MEE); mientras que una última categoría toma como foco el componente cognitivo, encontrándose los instrumentos incluidos en ella datados en fechas muy anteriores a las de las dos primeras categorías (DRTIE en 1949 y EM en 1969). En lo que se refiere a su estructura, todos estos instrumentos constan de un número de subescalas que oscila entre 2 y 7.

En los más recientes -IRI, EQ y TECA-, se observa que las dimensiones analizadas adoptan denominaciones similares, comenzando por el Índice de Reactividad Interpersonal (IRI) (Davis, 1980, 1983) que, de acuerdo con Mestre Escrivá, Frías Navarro, y Samper García (2004), es uno de los más utilizados. Sus 4 subescalas incluyen factores cognitivos y emocionales: Toma de perspectiva(PT), Fantasía (FS), Preocupación empática (EC) y Malestar personal (PD). El Cociente de Empatía (EQ) de Baron-Cohen y Wheelwright (2004) también integra la perspectiva multidimensional con dos subescalas denominadas Cognitiva y Reactividad emocional. A estas dos se añade una más, etiquetada Habilidades sociales. Para finalizar, y también desde una aproximación integradora, se ha desarrollado para la población de habla hispana el Test de Empatía Cognitiva y Afectiva (TECA) (López-Pérez, Fernández-Pinto \& Abad, 2008), que contempla, dentro de las subescalas cognitivas, la Comprensión Emocional. Esta subescala pretende fusionar ambas esferas, la cognitiva y la emocional.
En definitiva, en los instrumentos se observa una tendencia a la medición de ambas dimensiones, afectiva y cognitiva, frente a las pruebas anteriores, que parecían centrarse en una sola de ellas. En cualquier caso, resulta evidente que no hay consenso en la medición de la empatía, y esto se encuentra en consonancia con lo que ya se ha afirmado anteriormente en relación con la delimitación del constructo (Fernández-Pinto et al., 2008; Gerdes et al., 2010).

Partiendo de esta premisa y considerando el ámbito de práctica que focaliza nuestro interés -la educación intercultural-, parece conveniente afrontar el diseño de una medida de calidad de la empatía que pueda ser utilizada en contextos multiculturales, justificándose esta necesidad en la capacidad predictora que este constructo posee en relación con la estereotipia y el prejuicio en ámbitos de diversidad cultural (González, 2011; González, Álvarez \& Fernández, 2012; Shih, Stotzer \& Gutierrez, 2013). Considerando el peso de los distintos componentes del sesgo intergrupal (en la estereotipia dominan las creencias, mientras que en el prejuicio pesa mucho más la faceta afectiva), así como su relación con las estrategias de control del sesgo ensayadas en experimentos anteriores y las características del sesgo intergrupal en la población española (Álvarez, 2005; González, 2011; Álvarez, Palmero \& Jiménez, 2011; Álvarez, Jiménez, Palmero \& González, 2014), se juzga necesaria una aproximación orientada al componente afectivo de la empatía y a su medida en contextos multiculturales en los que el sesgo hacia distintos exogrupos es desigual.

\section{Empatía intercultural}

Aunque la empatía haya sido objeto de numerosas investigaciones desde diferentes perspectivas, cuando se trata de analizarla desde un enfoque cultural o étnico, deviene en un campo escasamente explorado (Green, 1998; Dyche \& Zayas, 2001; Rasoal, Eklund \& Hansen, 2011), en el que el constructo tampoco ha sido etiquetado ni operacionalizado de la misma forma. 
Tal vez sean Ridley y Lingle (1996) los primeros investigadores que usan y definen el concepto de empatía cultural, que sobrepasaría al de empatía general, incluyendo la comprensión y la aceptación de la cultura del otro. Estos autores argumentan que la cultura marca diferencias a menudo relevantes en valores y expectativas, de tal forma que la respuesta empática implica la comprensión mutua en relación con estas diferencias culturales. Asimismo, también se ha evidenciado que la misma empatía se encuentra asociada a diferencias culturales. Es decir, el nivel de regulación emocional y de interés empático por otra persona varían en función de la cultura de pertenencia (Trommsdorf, Friedlmeier \& Mayer, 2007), lo que validaría asimismo el concepto de empatía cultural. No obstante, a pesar de la potencialidad de este tipo contextualizado de empatía, la investigación sobre el mismo ha sido escasa (Howe, 2013).

Por su parte, Wang et al. (2003), conscientes del valor de los componentes culturales y étnicos, desarrollaron el concepto de empatía etnocultural, similar al de empatía cultural y asimismo afín a los de competencia cultural y empatía transcultural (Green, 1998; Lawrence \& Luis, 2001; Wang et al., 2003). Wang y sus colaboradores entendieron la empatía etnocultural como aquella mostrada hacia miembros de grupos culturales, étnicos y raciales que son diferentes al propio. El progreso en este tipo de empatía implicaría el retroceso de la intolerancia, la discriminación y los conflictos, al tiempo que avanzaría la comprensión y el respeto mutuo por los demás, independientemente de su grupo cultural o étnico de pertenencia. Se trata de una capacidad más difícil de desarrollar que la mera empatía interpersonal, ya que supone, de entrada, la asunción de la perspectiva de alguien que es percibido como miembro exogrupal. Luego el otro debe ser percibido en su contexto cultural para poder adoptar su perspectiva -este sería un primer rasgo diferencial de la empatía etnocultural-. En segundo lugar, la empatía etnocultural implica el control de los propios prejuicios hacia el grupo étnico o cultural al que pertenece la persona con la que se empatiza. Por último, el tercer rasgo distintivo de este tipo de capacidad empática es que ésta no es independiente de la experiencia previa con la otra cultura.

A partir de esta conceptuación, Wang et al. (2003) desarrollaron una escala de empatía etnocultural cuya validez y fiabilidad todavía son objeto de estudio, llegando a identificar los siguientes factores: expresión y sentimiento empático, toma de perspectiva empática, aceptación de las diferencias culturales y conciencia empática. A este respecto Albiero y Matricardi (2013), además de confirmar una validez y fiabilidad adecuadas de la escala diseñada por Wang et al. (2003) mediante métodos de análisis multivariante, encontraron una asociación moderada con la escala de empatía general y una fuerte relación negativa con la medida de prejuicio. Este tipo de vínculos actitudinales confirman los verificados en otros estudios entre la empatía general y las actitudes de prejuicio (Batson et al., 1997; Shih et al., 2009, 2013).

Tomando este instrumento como referente, se abordó la validación de una medida capaz de evaluar específicamente la empatía intercultural, que pudiera ser utilizada en contextos educativos diversos, de rápida implementación y fácil interpretación, considerando además la particularidad del contexto español.

Desde el enfoque intercultural tiene sentido que las medidas se encuentren asociadas a los contextos socioculturales en las que se aplican, ya que éstos marcan diferencias relevantes en los niveles de empatía y en la capacidad predictiva de esta variable (Howe, 2013). Por este motivo, en nuestro estudio se ha seleccionado, como grupo objeto de empatía en el instrumento a diseñar, el colectivo marroquí. Este representa la minoría nacional no europea más numerosa en España, resultando ser la que el grupo poblacional mayoritario vincula en mayor medida con el fenómeno de la inmigración que, a su vez, se asocia a estereotipos y prejuicios. Así, a la 
pregunta que formuló el Centro de Investigaciones Sociológicas (CIS, 2014) acerca del primer colectivo en que piensan los españoles cuando se habla de inmigrantes, los marroquíes fueron mencionados en primer lugar. También ocuparon un puesto elevado en lo que se refiere a inmigrantes que caen peor o por los que se tiene menos simpatía (superados en este caso únicamente por el colectivo rumano). Considerando este hecho, así como la relación ya mencionada de la empatía con el prejuicio, se presentará a continuación el método conducente al desarrollo de un instrumento de medición de la empatía hacia el colectivo marroquí, incidiendo sobre todo en su componente afectivo.

\section{Método}

\section{Muestra}

La investigación basada en encuesta incluyó dos muestras con la finalidad de realizar una validación cruzada: la primera para implementar el estudio exploratorio, y la segunda para el confirmatorio. En total participaron en ambos estudios 821 estudiantes universitarios de titulaciones pedagógicas (títulos en extinción de Diplomado en Educación Social y Licenciatura de Psicopedagogía, y Grados de Educación Primaria y Educación infantil), seleccionados de manera incidental. Las muestras, tanto exploratoria como confirmatoria, pertenecían en una proporción semejante a las provincias de Córdoba (48.3\%) y Burgos (51.7\%). Ambas provincias fueron seleccionadas por representar contextos culturales distintos y tener una representación desigual del colectivo marroquí, lo que podía marcar diferencias en empatía intercultural. En concreto, la población marroquí representa el $0.35 \%$ de la población cordobesa y el $0.75 \%$ de la burgalesa (INE, 2015). Sin embargo, no pudieron verificarse diferencias significativas en ninguna de las dimensiones de empatía intercultural entre ambos contextos, tal como se observa más adelante en la tabla 1 sobre el total de los participantes, antes de detraer la mortalidad derivada de los análisis que posteriormente se describirán. Esta invarianza facilitó el análisis conjunto de los datos.

La primera de las muestras, de carácter piloto, se empleó para comprobar el índice de discriminación de los ítems y analizar la estructura factorial del instrumento. Estuvo integrada por 250 alumnos/as de titulaciones pedagógicas de las Universidades de Córdoba y Burgos. El 73.2\% fueron mujeres y el 26.8\% restante, hombres. La media de edad de este grupo fue de 22 años con una desviación típica de 4.46 .

Por su parte, la muestra de participantes invitada para el estudio confirmatorio constó de 571 personas, de las cuales el $69.9 \%$ eran mujeres y el $30.1 \%$ eran varones. La media de edad del total de la muestra fue de 22.2 años con una desviación típica de 4.81.

\section{Instrumento}

El instrumento fue diseñado ad hoc conforme a las inferencias derivadas del marco teórico, y de acuerdo con las indicaciones de Zhou, Valiente y Eisenberg (2003) en su revisión acerca de los distintos métodos utilizados para evaluar la empatía (véase también Batson, 1987). De este modo, se optó por medir el componente evaluativo del sesgo hacia miembros de un colectivo concreto, identificados a través de imágenes que se presentaban en una pantalla de ordenador.

Se optó por el software Direct RT de Empirisoft para recoger las respuestas de los participantes a 20 ítems críticos, precedidos de unas instrucciones y una serie de preguntas de carácter sociodemográfico: edad, sexo y algunos ítems dirigidos a excluir a los participantes del análisis en caso de respuesta positiva (existencia de relación con personas de origen inmigrante en cualquier esfera: familiares, pareja, vecinos, compañeros de clase 0 equipo en alguna actividad extraacadémica; así como frecuencia $y$ duración de dichos contactos).

En el instrumento se pedía a los participantes, en cada uno de los 20 ítems, que evaluaran el grado en que experimentaban determinadas emociones mientras observaban 
una fotografía en una pantalla de ordenador. Las imágenes representaban rostros prototípicos de miembros del exogrupo (inmigrantes marroquíes). Las 20 imágenes fueron seleccionadas a partir de las puntuaciones de prototipicismo que 4 jueces otorgaron a 100 fotografías de un banco de rostros de personas inmigrantes procedentes de Marruecos. Previamente, las fotografías habían sido filtradas para unificar el formato: tamaño, color, luminosidad y ángulo. Los jueces, 2 profesores de la Universidad de Córdoba y 2 de la Universidad de Burgos, fueron seleccionados por su experiencia en el campo de la educación intercultural, pidiéndoseles que puntuaran el tipicismo de las personas mostradas con respecto a la categoría "marroquí" en una escala de 5 niveles $(\mathrm{A}=$ Aspecto idéntico al de una persona marroquí; $\mathrm{B}=$ aspecto parecido al de una persona marroquí; $\mathrm{C}=$ Aspecto indefinido; $\mathrm{D}=$ Aspecto algo diferente al de una persona marroquí; $\mathrm{E}=$ Aspecto totalmente diferente al de una persona marroquí). Se descartaron las fotos en las que no hubo un consenso total de los jueces en el nivel A y, entre las que reunían este requisito, fueron seleccionadas las de mejor calidad o definición.

Las emociones autoevaluadas fueron cinco: conmoción, comprensión, ternura, afecto y compasión (véase Batson, 1991; Vescio, Sechrist \& Paolucci, 2003), presentándosele al participante cuatro imágenes por cada una de las emociones sobre las que se le encuestaba. La escala de respuesta adoptó un formato tipo Likert de 7 puntos correspondientes al grado en que el encuestado decía experimentar la emoción que leía en la etiqueta situada debajo de cada imagen $(1=$ "Nada en absoluto", 7 = “Extremadamente”). El orden de presentación de las imágenes fue aleatorio para cada participante, neutralizándose así posibles sesgos debidos a la posición de la imagen en la secuencia de ítems.

\section{¿En qué medida te inspira COMPRENSIŌN la persona marroqui de la imagen?}
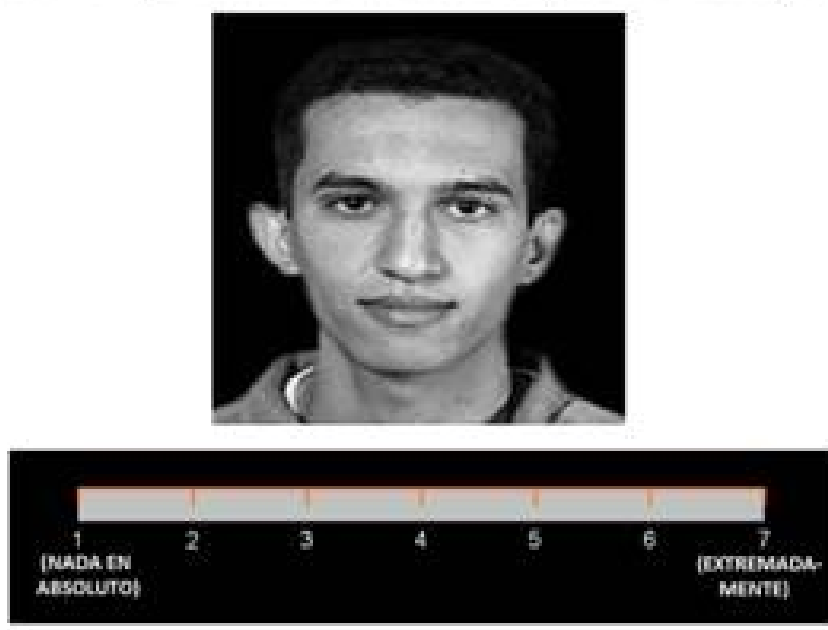

Figura 1. Ejemplo de ítem del test

\section{Análisis de datos}

Una vez descartados los participantes que, de acuerdo a sus respuestas al cuestionario sociodemográfico, pudieran estar emocionalmente comprometidos con el colectivo de personas inmigrantes (8 en el análisis exploratorio), se procedió a normalizar la muestra. Para ello se eliminaron los outliers detectados mediante el test de Mahalanobis, haciéndose esto con ayuda del software AMOS 22.0. De este modo, la muestra exploratoria contó finalmente con 211 participantes, 154 mujeres y 57 hombres con una media de edad de 21.80 y una desviación típica de 4.27. 
En el análisis exploratorio se procedió con el SPSS 22.0 a calcular los estadísticos descriptivos de la muestra, analizándose asimismo las propiedades de los ítems. Se comprobó que la normalidad univariante y multivariante de la distribución mostraba unos parámetros adecuados (Byrne, 2012; González, Abad \& Lévy, 2006; Kline, 2011). A continuación, se procedió a realizar el análisis factorial exploratorio mediante el programa FACTOR (Lorenzo-Seva \& Ferrando, 2006) que nos permitió llevar a cabo análisis mediante matrices de correlación policóricas, empleándose PA-MBS como procedimiento para determinar el número de factores, MRFA como método de extracción de factores y Promin como método de rotación.

En cuanto al análisis confirmatorio, se procedió en primer lugar a descartar los 17 participantes de la segunda muestra que mantenían algún lazo afectivo con distintos colectivos de personas inmigrantes. Del mismo modo, se eliminaron los outliers de la muestra mediante el test de Mahalanobis (AMOS 22.0), lo que redujo su tamaño a 482 participantes, 149 hombres y 333 mujeres con una media de edad de 22.08 y desviación típica de 4.76. Se utilizaron modelos de ecuaciones estructurales con el programa AMOS 22.0, evaluándose el ajuste del modelo mediante los estadísticos de bondad de ajuste que la mayoría de los autores defienden. Estos serían, según Byrne (2001), los siguientes: la prueba $\chi^{2}$ y la razón $\chi^{2}$ /grados de libertad, el índice de bondad de ajuste comparativo (CFI) (Bentler, 1990), el índice de bondad de ajuste general (GFI) y el índice de bondad de ajuste corregida (AGFI), el índice de Tuker-Lewis
(TLI), la raíz del residuo cuadrático promedio (RMR), la raíz del residuo cuadrático promedio de aproximación (RMSEA), el criterio de información de Aiken (AIC) y el índice de validación cruzada esperada (ECVI).

A continuación se obtuvo la fiabilidad de cada una de las dimensiones mediante el coeficiente alfa de Cronbach. Así mismo, para analizar la validez y fiabilidad del instrumento se calcularon, con ayuda del software AMOS 22.0, los índices recomendados en la literatura: Fiabilidad Compuesta (CR), Varianza Promedio Extraída (AVE), Varianza Máxima Compartida al Cuadrado (MSV) y Varianza Promedio Compartida al Cuadrado (ASV). Con ayuda de estos índices se establecieron la fiabilidad, la validez convergente y la validez discriminante.

Finalmente, sobre la muestra total, utilizando el SPSS y AMOS 22.0, se llevó a cabo un estudio sobre las diferencias de género en las diferentes dimensiones que componían el cuestionario validado.

\section{Resultados}

En primer lugar, como puede observarse en la tabla 1, las medias globales son indicativas de niveles medios de empatía con tendencia a medio-altos en los cinco factores iniciales. Asimismo, se comprueba que los participantes de las dos procedencias geográficas -a pesar de las supuestas diferencias contextuales- experimentaron las cinco emociones empáticas con una intensidad equivalente. Del mismo modo, las medias en las reacciones emocionales tampoco difirieron entre las muestras de ambos estudios (exploratorio y confirmatorio). 
González-González, Hugo; Álvarez-Castillo, José-Luis \& Fernández-Caminero, Gemma (2015). Desarrollo y validación de una escala de medida de la empatía intercultural. RELIEVE, 21 (2), art. 3. DOI: http://dx.doi.org/10.7203/relieve.21.2.7841

Tabla 1. Estadísticos descriptivos de las cinco dimensiones iniciales por muestras parciales y muestra global inicial ( $\mathrm{n}=821$ ), y pruebas de hipótesis sobre diferencias de medias entre los participantes de ambas provincias y estudios

\begin{tabular}{|c|c|c|c|c|c|c|c|c|c|c|c|c|}
\hline Dimensión & $\begin{array}{l}\text { Muestra/ } \\
\text { residencia }\end{array}$ & Media & $\begin{array}{c}\text { Desviación } \\
\text { Típica }\end{array}$ & $t(\mathbf{8 1 9 )}$ & $p$ & $\begin{array}{l}\text { Muestra/ } \\
\text { estudio }\end{array}$ & Media & $\begin{array}{c}\text { Desviación } \\
\text { Típica }\end{array}$ & $t(81$ & $p$ & $\begin{array}{l}\text { Media } \\
\text { global }\end{array}$ & $\begin{array}{c}\text { Desviaciór } \\
\text { típica } \\
\text { global }\end{array}$ \\
\hline \multirow{2}{*}{ Comprensión } & Burgos & 5.099 & 1.105 & \multirow{2}{*}{0.330} & \multirow{2}{*}{.741} & Exploratorio & 5.158 & 1.155 & \multirow{2}{*}{1.147} & \multirow{2}{*}{.252} & \multirow{2}{*}{5.087} & \multirow[b]{2}{*}{1.167} \\
\hline & Córdoba & 5.072 & 1.241 & & & Confirmatorio & 5.056 & 1.172 & & & & \\
\hline \multirow[b]{2}{*}{ Compasión } & Burgos & 4.618 & 1.216 & \multirow[b]{2}{*}{-1.042} & \multirow[b]{2}{*}{.298} & Exploratorio & 4.598 & 1.298 & \multirow[b]{2}{*}{-.929} & \multirow[b]{2}{*}{.353} & \multirow[b]{2}{*}{4.661} & \multirow[b]{2}{*}{1.300} \\
\hline & Córdoba & 4.714 & 1.396 & & & Confirmatorio & 4.689 & 1.301 & & & & \\
\hline \multirow[b]{2}{*}{ Conmoción } & Burgos & 4.648 & 1.208 & \multirow[b]{2}{*}{-1.644} & \multirow[b]{2}{*}{.100} & Exploratorio & 4.698 & 1.175 & \multirow[b]{2}{*}{-.190} & \multirow[b]{2}{*}{.849} & \multirow[b]{2}{*}{4.710} & \multirow[b]{2}{*}{1.205} \\
\hline & Córdoba & 4.786 & 1.198 & & & Confirmatorio & 4.715 & 1.219 & & & & \\
\hline \multirow[b]{2}{*}{ Ternura } & Burgos & 4.622 & 1.284 & \multirow[b]{2}{*}{-1.258} & \multirow[b]{2}{*}{.209} & Exploratorio & 4.767 & 1.287 & \multirow[b]{2}{*}{1.308} & \multirow[b]{2}{*}{.191} & \multirow[b]{2}{*}{4.675} & \multirow[b]{2}{*}{1.332} \\
\hline & Córdoba & 4.739 & 1.387 & & & Confirmatorio & 4.634 & 1.350 & & & & \\
\hline \multirow{2}{*}{ Afecto } & Burgos & 4.647 & 1.243 & \multirow{2}{*}{-1.518} & \multirow{2}{*}{.129} & Exploratorio & 4.748 & 1.191 & \multirow{2}{*}{.635} & \multirow{2}{*}{.526} & \multirow{2}{*}{4.706} & \multirow{2}{*}{1.240} \\
\hline & Córdoba & 4.779 & 1.233 & & & Confirmatorio & 4.688 & 1.261 & & & & \\
\hline
\end{tabular}

Mediante el análisis factorial exploratorio se comprobó, respecto a la estructura, que el modelo de 5 factores mostraba unos valores adecuados (tablas 2 y 3). La saturación de los ítems en sus factores teóricos superó ampliamente el umbral de .30. Así mismo, los índices de discriminación de los ítems (por medio de la correlación ítem-total corregida, obtenida con ViSta-CITA) y la fiabilidad estimada de los factores (tabla 4) resultaron ser de un tamaño elevado. Por otra parte, la correlación entre factores (ninguna alcanza .70) permite anticipar que la estructura factorial es adecuada. De esta forma, el inventario quedó provisionalmente constituido tal como se planteó inicialmente: 20 ítems correspondientes a 5 factores con 4 ítems cada uno.

Tabla 2. Pesos factoriales de los ítems en cada factor y correlaciones ítem-total corregidas

\section{Comprensión Compasión Conmoción Ternura Afecto Correlación Item-} Total Corregida

\begin{tabular}{ccccccc} 
Ítem & & & & & & \\
1 & .635 & -.004 & -.051 &.- .080 & .184 & .550 \\
2 & .732 & .009 & -.005 & .012 & .079 & .603 \\
3 & .900 & .014 & -.042 & .028 & -.118 & .551 \\
4 & .718 & -.182 & .020 & .019 & .166 & .548 \\
5 & .147 & .758 & .071 & -.147 & -.095 & .565 \\
6 & .016 & .682 & .131 & -.079 & .065 & .650 \\
7 & .020 & .773 & -.039 & .047 & .033 & .665 \\
8 & -.065 & .752 & -.112 & .224 & .008 & .611 \\
9 & .043 & .062 & -.039 & .034 & .630 & .565 \\
10 & .063 & .044 & -.103 & -.059 & .812 & .571 \\
11 & -.048 & -.066 & .119 & .022 & .699 & .591 \\
12 & .021 & -.050 & .080 & .060 & .569 & .558 \\
13 & -.169 & -.004 & -.081 & .740 & .221 & .482 \\
14 & .025 & -.079 & .091 & .774 & -.094 & .566 \\
15 & .102 & .012 & .025 & .674 & -.048 & .529 \\
16 & .111 & .098 & .016 & .573 & -.062 & .529 \\
17 & .037 & -.063 & .819 & .041 & -.083 & .620 \\
18 & .053 & .105 & .687 & -.122 & .070 & .643 \\
19 & -.073 & .051 & .662 & -.053 & .157 & .641 \\
20 & -.049 & -.078 & .701 & .174 & -.090 & .629 \\
Varianza explicada & $\mathbf{1 8 . 4}$ & $\mathbf{1 7 . 3}$ & $\mathbf{1 6 . 4}$ & $\mathbf{1 5 . 8}$ & $\mathbf{1 6 . 6}$ & \\
\hline
\end{tabular}


González-González, Hugo; Álvarez-Castillo, José-Luis \& Fernández-Caminero, Gemma (2015). Desarrollo y validación de una escala de medida de la empatía intercultural. RELIEVE, 21 (2), art. 3. DOI: http://dx.doi.org/10.7203/relieve.21.2.7841

Tabla 3. Matriz de correlaciones entre factores

\begin{tabular}{llllll}
\hline Factor & Comprensión & Compasión & Conmoción & Ternura & Afecto \\
\hline 1 Comprensión & 1.000 & & & & \\
2 Compasión & .476 & 1.000 & & & \\
3 Conmoción & .564 & .652 & 1.000 & & \\
4 Ternura & .599 & .439 & .576 & 1.000 & \\
5 Afecto & .599 & .495 & .659 & .607 & 1.000 \\
\hline
\end{tabular}

Conjuntamente, los 5 factores explicaron el $84.48 \%$ de la varianza total. Se obtuvo una media de los residuales de -0.002 y una varianza de los mismos de 0.001 . La raíz del residuo cuadrático promedio (RMSR) del modelo fue 0.028 , muy por debajo del valor medio esperado de RMSR (0.069) para un modelo aceptable, de acuerdo con el criterio de Kelley (1935; véase también Harman, 1962).

Sin embargo, al comenzar el análisis factorial confirmatorio sobre los datos de la segunda muestra, los índices de modificación señalaban la existencia de covarianzas entre errores asociados a ítems pertenecientes a distintos factores. Esta circunstancia propició que el modelo se reformulara, tal como puede observarse en la figura 2, pasando de los 5 factores iniciales a sólo 3 factores (tres de los factores iniciales se resumieron en uno más general: «Preocupación empática»). Por otra parte, tras comprobar las matrices de covarianza de los residuales estandarizados, en los tres factores analizados se observó que todos los valores eran inferiores a 2.58. Sólo una intersección de ítems (Ternura_A $\mathrm{x}$ Comprensión_D) se acercaba al límite mencionado con un valor de 2.54, por lo que se procedió eliminar el ítem Comprensión_D y también el ítem Ternura_B, que tenía dos valores inferiores al citado, pero también próximos al límite establecido. Este dato es otro indicador de un buen ajuste de los modelos (Joreskog y Sorbom, 1988; Byrne, 2001). 


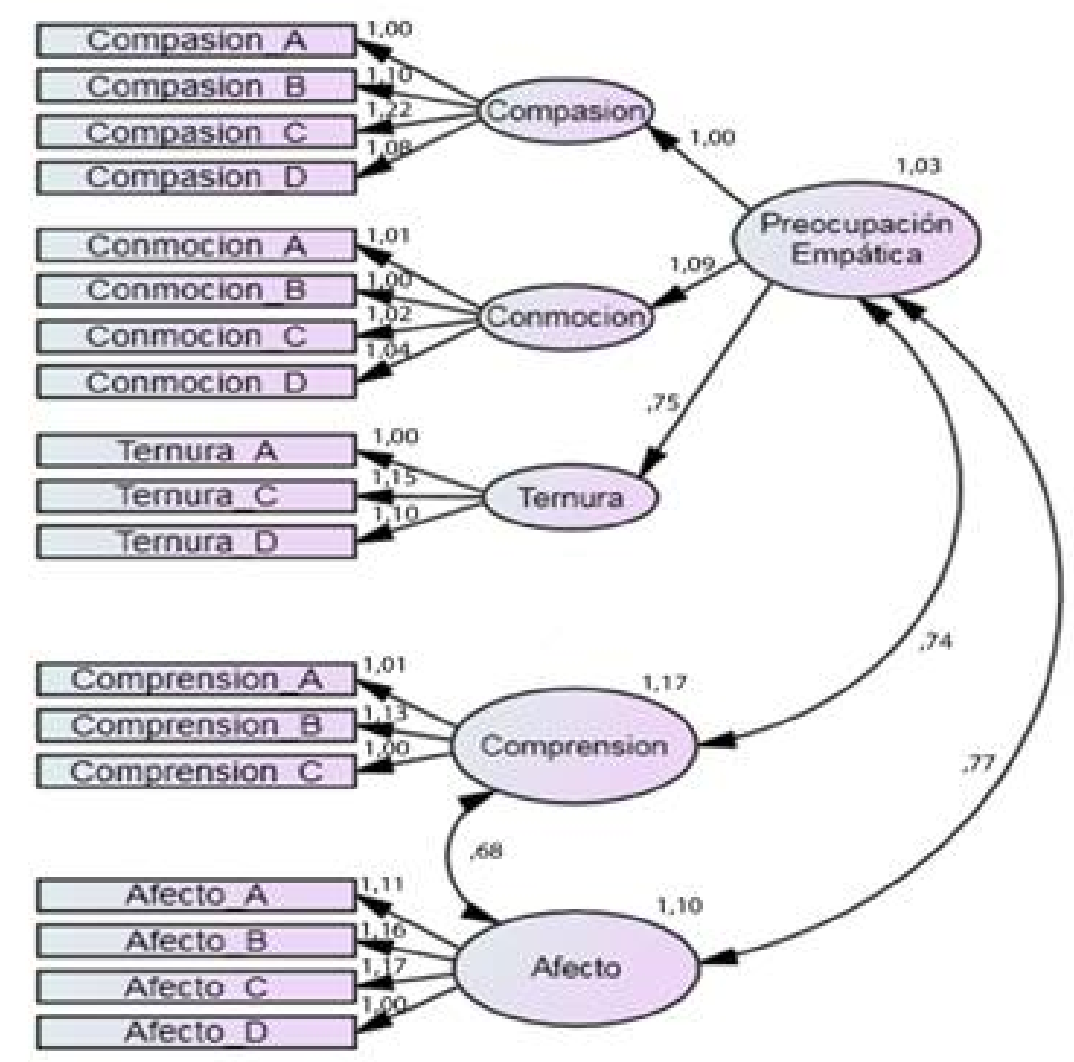

Figura 2. Modelo de estructura factorial de segundo orden del cuestionario de empatía intercultural hispano-marroquí, y estimaciones estandarizadas de los pesos de regresión.

Los coeficientes de fiabilidad de los factores y de la prueba, tal como muestra la tabla 4, apenas sufrieron cambios. Aun así, para confirmar la adecuación del nuevo modelo con 3 factores, se procedió de nuevo a calcular los estadísticos correspondientes al análisis exploratorio, pero forzando en esta ocasión un modelo con 3 factores, arrojando resultados igualmente favorables en varianza explicada, índice de discriminación de los ítems, fiabilidad estimada de los factores y del conjunto de la prueba. Aunque la varianza total explicada por las técnicas factoriales se redujo al $74.84 \%$, la fiabilidad de cada factor se mantuvo por encima de .80 (tabla 4) y la validación del modelo utilizando análisis estructurales resultó, como puede apreciarse en la tabla 5, ampliamente satisfactoria.

Tabla 4. Coeficientes de fiabilidad de los factores y del conjunto de la prueba, en los modelos de 5, 4 y 3 factores

\begin{tabular}{|c|c|c|c|c|c|c|c|c|}
\hline 5 factores & $\begin{array}{l}\mathrm{N}^{0} \text { de } \\
\text { ítems }\end{array}$ & $\alpha$ & 4 factores & $\begin{array}{l}\mathrm{N}^{0} \text { de } \\
\text { ítems }\end{array}$ & $\alpha$ & 3 factores & $\begin{array}{l}\mathrm{N}^{\circ} \text { de } \\
\text { ítems }\end{array}$ & $\alpha$ \\
\hline Afecto & 4 & 0.809 & Afecto & 4 & 0.809 & Afecto & 4 & 0.870 \\
\hline Comprensión & 4 & 0.823 & Comprensión & 4 & 0.823 & Comprensión & 3 & 0.910 \\
\hline Ternura & 4 & 0.776 & Ternura & 4 & 0.776 & Preocupación E. & 11 & 0.864 \\
\hline Compasión & 4 & 0.860 & Preocupación E. & 8 & 0.895 & Total & 18 & 0.923 \\
\hline Conmoción & 4 & 0.839 & Total & 20 & 0.922 & & & \\
\hline Total & 20 & 0.922 & & & & & & \\
\hline
\end{tabular}


González-González, Hugo; Álvarez-Castillo, José-Luis \& Fernández-Caminero, Gemma (2015). Desarrollo y validación de una escala de medida de la empatía intercultural. RELIEVE, 21 (2), art. 3. DOI: http://dx.doi.org/10.7203/relieve.21.2.7841

Tabla 5. Índices de ajuste del modelo de tres factores

\begin{tabular}{lcccccccccccc}
\hline & $\chi^{2}$ & $\boldsymbol{d} \boldsymbol{f}$ & $\boldsymbol{p}$ & $\boldsymbol{X}^{2} / \boldsymbol{d} \boldsymbol{f}$ & $\mathbf{G F I}$ & $\mathbf{C F I}$ & $\begin{array}{c}\text { NNFI } \\
\mathbf{( T L I )}\end{array}$ & SRMR & RMSEA & $\begin{array}{c}\text { Superior } \\
\mathbf{( 9 0 \% )}\end{array}$ & $\begin{array}{c}\text { Inferior } \\
\mathbf{( 9 0 \% )}\end{array}$ & PCLOSE \\
\hline $\begin{array}{l}\text { Muestra } \\
\text { completa } \\
(\mathrm{n}=482)\end{array}$ & 470.28 & 303 & .000 & 1.55 & .950 & .978 & .967 & .0361 & .024 & .028 & .020 & 1.000 \\
\hline $\begin{array}{l}\text { Hombres } \\
(\mathrm{n}=149)\end{array}$ & 154.80 & 101 & .000 & 1.53 & .902 & .960 & .939 & .0672 & .060 & .078 & .040 & .187 \\
\hline $\begin{array}{l}\text { Mujeres } \\
(\mathrm{n}=333)\end{array}$ & 145.53 & 101 & .002 & 1.44 & .954 & .982 & .973 & .0351 & .036 & .049 & .022 & .962 \\
\hline
\end{tabular}

El modelo resultante cumple los requisitos de ajuste exigibles y mejora los índices de validez conseguidos con los modelos ensayados de cinco o cuatro factores. Concretamente, en la tabla 6 se presentan los coeficientes de validez y fiabilidad derivados del análisis de los pesos de regresión estandarizados y las correlaciones obtenidas con AMOS 22.0. Los resultados se pueden considerar más que aceptables, sobre todo teniendo en cuenta el bajo número de ítems que componen cada factor. Respecto al debate sobre la multidimensionalidad o unidimensionalidad de la empatía, se asume que las respuestas al cuestionario son descritas de una forma más adecuada por una estructura factorial jerárquica. Es decir, tres de los factores de primer orden (Compasión, Conmoción y Ternura) son explicados por un único factor de segundo orden que se ha denominado «Preocupación Empática», tal y como aparece en el modelo representado en la figura 2.

Tabla 6. Coeficientes de validez y fiabilidad de los modelos de 5, 4 y 3 factores

\begin{tabular}{|c|c|c|c|c|c|c|c|}
\hline & CR & AVE & MSV & ASV & $\begin{array}{c}\text { Fiabilidad } \\
\text { CR }>.7\end{array}$ & $\begin{array}{c}\text { Validez Convergente } \\
\text { CR }>\text { AVE, } \\
\text { AVE }>.5\end{array}$ & $\begin{array}{c}\text { Validez } \\
\text { Discriminante } \\
\text { MSV }<\text { AVE, } \\
\text { ASV }<\text { AVE }\end{array}$ \\
\hline \multicolumn{8}{|c|}{ Modelo de 5 factores } \\
\hline Comprensión & 0.82 & 0.60 & 0.37 & 0.35 & $\checkmark$ & $\checkmark$ & $\checkmark$ \\
\hline Afecto & 0.82 & 0.54 & 0.45 & 0.40 & $\checkmark$ & $\checkmark$ & $\checkmark$ \\
\hline Compasión & 0.83 & 0.56 & 0.82 & 0.46 & $\checkmark$ & $\checkmark$ & $\mathbf{X}$ \\
\hline Conmoción & 0.82 & 0.52 & 0.82 & 0.50 & $\checkmark$ & $\checkmark$ & $\mathbf{X}$ \\
\hline Ternura & 0.71 & 0.45 & 0.43 & 0.37 & $\checkmark$ & $\mathbf{X}$ & $\checkmark$ \\
\hline \multicolumn{8}{|c|}{ Modelo de 4 factores } \\
\hline Comprensión & 0.80 & 0.57 & 0.42 & 0.38 & $\checkmark$ & $\checkmark$ & $\checkmark$ \\
\hline Afecto & 0.83 & 0.55 & 0.47 & 0.43 & $\checkmark$ & $\checkmark$ & $\checkmark$ \\
\hline Preocupación E. & 0.93 & 0.87 & 0.47 & 0.41 & $\checkmark$ & $\checkmark$ & $\checkmark$ \\
\hline Ternura & 0.74 & 0.48 & 0.45 & 0.43 & $\checkmark$ & $\mathbf{X}$ & $\checkmark$ \\
\hline \multicolumn{8}{|c|}{ Modelo de 3 factores } \\
\hline Comprensión & 0.82 & 0.60 & 0.45 & 0.40 & $\checkmark$ & $\checkmark$ & $\checkmark$ \\
\hline Afecto & 0.83 & 0.54 & 0.52 & 0.44 & $\checkmark$ & $\checkmark$ & $\checkmark$ \\
\hline $\begin{array}{l}\text { Preocupación } \\
\text { Empática }\end{array}$ & 0.89 & 0.72 & 0.52 & 0.49 & $\checkmark$ & $\checkmark$ & $\checkmark$ \\
\hline
\end{tabular}


Respecto a las diferencias de género, se comprueba en la tabla 7 que las mujeres obtienen puntuaciones significativamente superiores en la subescala de comprensión y en la de preocupación empática.

Tabla 7. Diferencias por género en las dimensiones del cuestionario

\begin{tabular}{|c|c|c|c|c|c|c|c|}
\hline & \multicolumn{2}{|c|}{ Media } & \multicolumn{2}{|c|}{ Desviación Típica } & \multirow[b]{2}{*}{$t$} & \multirow[b]{2}{*}{$g l$} & \multirow[b]{2}{*}{$p$} \\
\hline & Hombres & Mujeres & Hombres & Mujeres & & & \\
\hline Comprensión & 4.85 & 5.19 & 1.22 & 1.24 & -2.745 & 480 & .006 \\
\hline Afecto* & 4.61 & 4.81 & 1.38 & 1.20 & -1.553 & 252.27 & .122 \\
\hline Preocupación Empática & 4.34 & 4.85 & 1.13 & 1.11 & -4.589 & 480 & $<.001$ \\
\hline
\end{tabular}

*En el factor "Afecto" no se asume igualdad de varianzas en ambos grupos ( $F=5.339, p=.021)$

\section{Discusión}

Frente a otros estudios que tratan de validar en una población determinada un instrumento generalista, desconsiderando la idiosincrasia propia del contexto en el que se aplica, la presente investigación ha validado una medida de empatía intercultural diseñada específicamente para medir esta variable en la población española en relación con un colectivo nacional minoritario muy relevante, como lo es el marroquí (CIS, 2014). A este respecto, los resultados muestran una validez y fiabilidad sólidas, así como una clara estructura factorial jerárquica que ha permitido agrupar dimensiones muy próximas conceptualmente.

La estrategia utilizada para el diseño de la medida validada, que se deriva de las observaciones detalladas en el marco teórico de este trabajo, se basa en la necesidad de construir herramientas que midan la dimensión emocional del sesgo mediante el uso, en el mismo instrumento, de imágenes u otros elementos que permitan al participante contextualizar sus sentimientos hacia el exogrupo. En este sentido, la medida diseñada supone un avance considerable con respecto a otras (e.g., Wang et al., 2003; véase también Albiero \& Matricardi, 2013) al recortar su longitud e introducir las imágenes como estímulo vívido y facilitador de respuestas semiautomáticas sobre miembros de grupos hacia los que se mantienen sólidos prejuicios en la población de referencia. En cuanto a la varianza explicada, mientras el análisis exploratorio del instrumento de Wang y sus colegas mantiene en su versión final 31 de los 62 ítems ensayados, que explican el $47 \%$ de la varianza, nuestro instrumento alcanza, con sólo 18 ítems, el 74.84\%. Por otra parte, el cuestionario publicado en 2003 utiliza un estudio de fiabilidad y de validación basada en test-retest, alfa de Cronbach y validez concurrente y discriminante mediante el análisis correlacional con otros instrumentos, sin aportar información acerca de los estadísticos de fiabilidad compuesta (CR), varianza promedio extraída (AVE), varianza máxima compartida al cuadrado (MSV) y varianza promedio compartida al cuadrado (ASV). A este respecto hay que señalar que resulta muy recomendable, cuando se realiza un análisis factorial confirmatorio, calcular la validez y fiabilidad mediante los estadísticos indicados. De otro modo, y a pesar de que se obtenga un ajuste del modelo satisfactorio, la fiabilidad y validez de la estructura de factores y sus ítems no queda demostrada. Por tanto, las variantes metodológicas pueden encontrarse en la base de las diferencias entre las estructuras factoriales arrojadas por el estudio de Wang et al. (2003) y por el nuestro, si bien tampoco debe descartarse el carácter modulador de la cultura a la que pertenecen las muestras de ambas investigaciones (Trommsdorff et al., 2007). Es decir, el adjetivo "intercultural” aplicado a la empatía haría alusión no sólo al colectivo que es objeto 
de reacción emocional, sino también a la interacción de los rasgos culturales del grupo perceptor con los del grupo percibido, y aquí reside la distintividad del constructo medido.

En lo que se refiere al género, cabe señalar que se han encontrado diferencias en dos de las tres dimensiones: comprensión y preocupación empática (esta última comprende tres factores de primer orden: compasión, conmoción y ternura). Estas diferencias muestran que las mujeres tienen niveles más altos de empatía intercultural. Sin embargo, en la tercera dimensión -afecto-, no se pudo rechazar la hipótesis nula. A este respecto, y aunque los datos vienen a corroborar esta diferencia de género (Hoffman, 1977; Lennon \& Eisenberg, 1987; Rueckert, Branch \& Doan, 2011), es interesante observar que Eisenberg y Lennon (1983) anticiparon que, en la medida de la empatía, este tipo de diferencias dependían del diseño del cuestionario. No obstante, estos autores afirmaban que los instrumentos que empleaban técnicas basadas en imágenes o historias mostraban menos diferencias por razón de género, mientras que aquellos cuestionarios basados en autoinformes distanciaban significativamente a hombres y mujeres a favor de estas últimas. Nuestra investigación apoyaría también, sin embargo, la existencia de diferencias en el caso del primer tipo de instrumentos.

Más allá de cuestiones relacionadas con el diseño de la investigación o el cuestionario, y aunque hay algunos estudios que afirman que las diferencias de género en empatía no son significativas (Block, 1979; Maccoby \& Jacklin, 1974; Rueckert et al., 2011) concluyen que se trata de un fenómeno robusto en la literatura, demostrando las mujeres niveles más elevados de empatía. Según estos autores, la diferencia de género puede ceñirse precisamente a la empatía de tipo emocional y se evidencia mediante los mayores niveles de reactividad emocional en mujeres. Los niveles de empatía en mujeres se ven más afectados por su relación con la otra persona. De este modo, mientras en su investigación se observaron mayores niveles de empatía hacia el colectivo de "amigos" que en sus pares varones, los niveles de empatía de las mujeres también fueron significativamente menores hacia personas consideradas "enemigos". Siendo esto así, cabe considerar que el colectivo de mujeres españolas perciba al exogrupo de origen marroquí menos amenazante de lo que lo hacen los hombres españoles.

Por otra parte, en esta cuestión del género resulta asimismo relevante la perspectiva evolutiva. Van der Graaff et al. (2014) revisaron en sus conclusiones, ante todo, dos teorías que justifican la diferencia por género en los niveles de empatía: las biológicas, basadas en las distintas maduraciones neurológicas o diferencias hormonales que son consecuencia de la maduración física, y las sociales, debidas a las expectativas derivadas de los distintos roles de género. De acuerdo con los resultados obtenidos por dichos autores, esta diferencia se acentúa durante la adolescencia y, según muestran nuestros datos, tal diferencia se mantendría y consolidaría después de dicha etapa.

En definitiva, tanto el instrumento obtenido como la evidencia resultante son de interés para futuras investigaciones orientadas a la medición de la empatía en contextos multiculturales y, si se considera la asociación consistente entre empatía y prejuicio (Batson et al., 1997; Shih et al., 2009, 2013), habrán de ser tomados en consideración en el diseño de estrategias pedagógicas encaminadas a la reducción o control del sesgo intergrupal en este tipo de entornos.

\section{Referencias}

Albiero, P. \& Matricardi, G. (2013). Empathy towards people of different race and ethnicity: Further empirical evidence for the Scale of Ethnocultural Empathy. International Journal of Intercultural Relations, 37, 648-655. doi: http://dx.doi.org10.1016/j.ijintrel.2013.05.003

Álvarez Castillo, J. L. (2005). Las metas en la reducción del prejuicio automático: 
experimentando la discrepancia. Psicothema, 17, 71-75.

Álvarez Castillo, J. L., Jiménez Eguizábal, A., Palmero Cámara, M. C. \& González González, H. (2014). The fight against prejudice in older adults: perspective taking effectiveness. Revista Latinoamericana de Psicología, 46, 137-147. doi: http://dx.doi.org/10.1016/S0120$\underline{0534(14) 70017-2}$

Álvarez Castillo, J.L., Palmero Cámara, M.C. \& Jiménez Eguizábal, A. (2011). Prejudice reduction in university programs for older adults. Educational Gerontology, 37, 164190. doi: http://dx.doi.org/10.1080/03601271003608811

Barnett, G. \& Mann, R. E. (2013). Empathy deficits and sexual offending: A model of obstacles to empathy. Aggression and Violent Behavior, 18, 228-239. doi: http://dx.doi.org/10.1016/j.avb.2012.11.010

Baron-Cohen, S. \& Wheelwright, S. (2004). The empathy quotient: An investigation of adults with Asperger syndrome or high functioning autism, and normal sex differences. Journal of Autism and Developmental Disorders, 34, 163-175. doi: http://dx.doi.org/10.1023/b:jadd.0000022607. $\underline{19833.00}$

Batson, C. D. (1987). Self-report ratings of empathic emotion. En N. Eisenberg \& J. Strayer (Eds.), Empathy and its development (pp. 356-360). New York: Cambridge University Press.

Batson, C. D. (1991). The altruism question: Toward a social-psychological answer. Hillsdale, NJ: Erlbaum.

Batson, C. D., Polycarpou, M. P., HarmonJones, E., Imhoff, H. J., Mitchner, E. C., Bednar, L. L., Klein, T. R. \& Highberger, L. (1997). Empathy and attitudes: Can feeling for a member of a stigmatized group improve feelings toward the group? Journal of Personality and Social Psychology, 72, 105118.
Belacchi, C. \& Farina, E. (2012). Feeling and Thinking of Others: Affective and Cognitive Empathy and Emotion Comprehension in Prosocial/Hostile Preschoolers. Aggressive Behavior, 38, 150-165. doi: http://dx.doi.org/10.1002/ab.21415

Bentler, P. M. (1990). Comparative fit indices in structural models. Psychological Bulletin, 107, 238-246.

Block, J. H. (1976). Assessing sex differences: Issues, problems, and pitfalls. Merrill Palmer Quarterly, 22, 283-308.

Butrus, N. \& Witenberg, R. T. (2013). Some Personality Predictors of Tolerance to Human Diversity: The Roles of Openness, Agreeableness, and Empathy. Australian Psychologist, 48, 290-298. doi: http://dx.doi.org/10.1111/j.17429544.2012.00081.x

Byrne, B. M. (2012). Structural Equation Modeling with Mplus: basic concepts, applications, and programming. New York: Taylor \& Francis Group.

Byrne, B.M. (2001). Structural Equation Modeling with AMOS. Basic Concepts, Applications, and Programming. Londres: LEA.

CIS (2014). Catálogo de encuestas. Inmigración. Recuperado de http://www.cis.es/cis/opencm/ES/1_encuesta s/estudios/listaTematico.jsp?tema=82\&todos $=$ no

Davis, M.H. (1980). A multidimensional Approach to Individual Differences in Empathy. JSAS Catalog of Selected Documents in Psychology, 10, 85.

Davis, M.H. (1983). Measuring Individual Differences in Empathy: Evidence for a Multidimensional Approach. Journal of Personality and Social Psychology, 44, 113126.

Dyche, L., \& Zayas, L. H. (2001). Crosscultural empathy and training the contemporary psychotherapist. Clinical 
Social Work Journal, 29, 245-258. doi: http://dx.doi.org10.1023/A:1010407728614

Eisenberg, N. (2000). Emotion, Regulation, and Moral Development. Annual Review of Psychology, 51, 665-697. doi: http://dx.doi.org/10.1146/annurev.psych.51.1 .665

Eisenberg, N. \& Lennon, R. (1983). Sex differences in empathy and related capacities. Psychological Bulletin, 94, 100-131.

Fernández-Pinto, I., López-Pérez, B. \& Márquez, M. (2008). Empatía: Medidas, teorías y aplicaciones en revisión. Anales de psicología, 2, 284-298. doi: http://dx.doi.org/10.6018/42831

Gerdes, K. E., Segal, E. A. \& Lietz, C. A. (2010). Conceptualising and measuring empathy. British Journal of Social Work, 40, 2326-2343. doi: http://dx.doi.org/10.1093/bjsw/bcq048

González González, H. (2011). La toma de perspectiva en el control del sesgo intergrupal: variables moduladoras de tipo individual y pautas pedagógicas. Universidad de Córdoba, Servicio de Publicaciones.

González González, H., Álvarez Castillo, J. L. \& Fernández Caminero, G. (2012). Fundamentando la formación de los educadores profesionales en competencias interculturales: la toma de perspectiva. Bordón, 64, 89-107.

Gónzalez, N., Abad, J. \& Lèvy, J.P. (2006). Normalidad y otros supuestos en análisis de covarianzas”. En J. P. Lévy (Ed.), Modelización con estructuras de covarianzas (pp. 31-57). La Coruña: Netbiblo.

Green, J. W. (1998). Cultural awareness in the human services: A multi-ethnic approach (3rd ed.). Toronto: Allyn \& Bacon.

Hardy, S. A., Walker, L. J., Rackham, D. D. \& Olsen, J. A. (2012). Religiosity and Adolescent Empathy and Aggression: The Mediating Role of Moral Identity. Psychology of Religion and Spirituality, 4,
237-248.

doi:

http://dx.doi.org/10.1037/a0027566

Harman, H. H. (1962). Modern Factor Analysis, 2nd Edition. University of Chicago Press, Chicago.

Harris, S. T. \& Picchioni, M. M. (2013). A review of the role of empathy in violence risk in mental disorders. Aggression and Violent Behavior, 18, 335-342. doi: http://dx.doi.org/10.1016/j.avb.2012.12.003

Hodges, S. D., Clark, B. A. M. \& Myers, M. W. (2011). Better living through perspective taking. En Biswas-Diener (Ed.), Positive psychology as social change (pp. 193-218). New York, NY: Springer Science + Business Media; US.

Hoffman, M. L. (1975). Developmental synthesis of affect and cognition and its implications for altruistic motivation. Developmental Psychology, 11, 607-622.

Hoffman, M. L. (1977). Personality and social development. Annual Review of Psychology, 28, 295-321.

Hoffman, M. L. (1981). The development of empathy. En J. Philippe Rushton \& Richard M. Sorrentino (Eds.), Altruism and helping behavior social, personality and developmental perspectives. New Jersey: L.E.A.

Hoffman, M. L. (1982). Development of prosocial motivation: Empathy and guilt. En N. Eisenberg (Ed.), The development of prosocial behavior. New York: Academic Press.

Hoffman, M. L. (1983). Empathy, guilt and social cognition. En W. F. Overton (Ed.), The relationship between social and cognitive development. London: L.E.A.

Howe, D. (2013). Empathy. What it is and why it matters. Basingstoke: Palgrave MacMillan.

INE (2015). INEbase: Operaciones estadísticas: clasificación por temas. Consultado en http://www.ine.es/inebmenu/indice.htm 
Joreskog, K. G. \& Sorbom, D. (1988). PRELIS: A program for multivariate data screening and data summarization. A preprocessor for LISREL ( $2^{\text {nd }}$ ed.). Mooresville, IN: Scientific Software.

Kelley, T. L. (1935). Essential Traits of Mental Life, Harvard Studies in Education, vol. 26. Harvard University Press, Cambridge.

Kline, R. B. (2011). Principles and Practice of Structural Equation Modeling (3rd Ed.). New York, The Guilford Press.

Lennon, R. \& Eisenberg, N. (1987). Gender and age differences in empathy and sympathy. En N. Eisenberg \& J. Strayer (Eds.), Empathy and its development (pp. 195-217). New York, NY: Cambridge University Press.

Li, W., Mai, X. \& Liu, C. (2014). The default mode network and social understanding of others: what do brain connectivity studies tell us. Frontiers in Human Neuroscience, 8, 115.

doi: http://dx.doi.org/10.3389/fnhum.2014.00074

López-Pérez, B., Fernández-Pinto, I. \& Abad, F. J. (2008). TECA. Test de Empatía Cognitiva y Afectiva. Madrid: Tea Ediciones, S.A.

Lorenzo-Seva, U. \& Ferrando, P.J. (2006). FACTOR: A computer program to fit the exploratory factor analysis model, Behavioral Research Methods, Instruments and Computers, 38, 88-91. doi: http://dx.doi.org/10.3758/BF03192753

Maccoby, E. E. \& Jacklin, C. N. (1974). The psychology of sex differences. Stanford: Stanford University Press.

Mardia, K. V. (1970). Measures of multivariate skewnees and kurtosis with applications. Biometrika, 57, 519-530

Mestre Escrivá, V., Frías Navarro, M. D. \& Samper García, P. (2004). La medida de la empatía: análisis del Interpersonal Reactivity Index. Psicothema, 16, 255-260.
Numata, J. (2013). Effects of a letter-writing task on empathy toward Japanese-Brazilians as out-group members: An educational method for intercultural understanding in universities and colleges. Psychologia, 56, 45-56. doi: http://dx.doi.org/10.2117/psysoc.2013.45

Peek, E. \& Park, C. (2013). Effects of a multicultural education program on the cultural competence, empathy and selfefficacy of nursing students. Journal of Korean Academy of Nursing, 43, 690-696. doi:

http://dx.doi.org/10.4040/jkan.2013.43.5.690

Rasoal, C., Eklund, J. \& Hansen, E. M. (2011). Toward a conceptualization of ethocultural empathy. Journal of Social, Evolutionary, and Cultural Psychology, 5, 1-13. doi: http://dx.doi.org/10.1037/h0099278

Ridley, C. R. \& Lingle, D.W. (1996). Cultural empathy in multicultural counseling: A multidimensional process model. En P. B. Pedersen \& J. G. Draguns (Eds.), Counseling across culture (4th ed., pp. 21-46). Thousand Oaks: CA: Sage.

Rueckert, L., Branch, B. \& Doan, T. (2011). Are Gender Differences in Empathy Due to Differences in Emotional Reactivity?. Psychology, 6, 574-578. doi: http://dx.doi.org/10.4236/psych.2011.26088

Sanmartín, M. G., Carbonell, A. E. \& Banos, C. P. (2011). Relationships among empathy, prosocial behavior, aggressiveness, selfefficacy and pupils' personal and social responsibility. Psicothema, 23, 13-19.

Shen, Y. L., Carlo, G. \& Knight, G. P. (2013). Relations between parental discipline, empathy-related traits, and prosocial moral reasoning: A multicultural examination. Journal of Early Adolescence, 33, 994-1021. doi:

http://dx.doi.org/10.1177/0272431613479670

Shih, M. J., Stotzer, R. \& Gutierrez, A. S. (2013). Perspective-Taking and Empathy: Generalizing the Reduction of Group Bias Towards Asian Americans to General 
Outgroups. Asian American Journal of Psychology, 4, 79-83. doi: http://dx.doi.org/10.1037/a0029790

Shih, M. J., Trahan, A., Wang, E. \& Stotzer, R. L. (2009). Perspective taking: Reducing prejudice towards general outgroups and specific individuals. Group Processes and Intergroup Relations, 12, 565-577. doi: http://dx.doi.org/10.1177/1368430209337463

Strayer, J. \& Eisenberg, N. (1987). Empathy viewed in context. En N. Eisenberg \& J. Strayer (Eds.), Empathy and its development (pp. 389-398). New York: Cambridge University Press.

Todd, A. R., Bodenhausen, G. V. \& Galinsky, A. D. (2012). Perspective taking combats the denial of intergroup discrimination. Journal of Experimental Social Psychology, 48, 738745. doi: http://dx.doi.org/10.1016/j.jesp.2011.12.011

Trommsdorff, G., Friedlmeier, W. \& Mayer, B. (2007). Sympathy, distress, and prosocial behavior of preschool children in four cultures. International Journal of Behavioural Development, 31(3), 284-293. doi:

http://dx.doi.org/10.1177/0165025407076441

Van der Graaff, J., Branje, S., De Wied, M., Hawk, S. \& Van Lier, P. (2014). Perspective taking and empathic concern in adolescence: gender differences in developmental changes. Developmental psychology, 3, 881-888. doi: http://dx.doi.org/10.1037/a0034325

Vescio, T. K., Sechrist, G. B. \& Paolucci, M. P. (2003). Perspective taking and prejudice reduction: The mediational role of empathy arousal and situational attribution. European Journal of Social Psychology, 33, 455-472. doi: http://dx.doi.org/10.1002/ejsp.163

Wang, Y.W., Davidson, M. M., Yakushko, O. F., Bielstein Savoy H., Tan, J. A. \& Bleier, J. K. (2003). The Scale of Ethnocultural Empathy: Development, Validation, and
Reliability. Journal of Counseling Psychology, 2, 221-234. doi: http://dx.doi.org/10.1037/0022$\underline{0167.50 .2 .221}$

Welp, L. R. \& Brown, C. M. (2014). Selfcompassion, empathy, and helping intentions. Journal of Positive Psychology, 9, 54-65. doi:

http://dx.doi.org/10.1080/17439760.2013.831 $\underline{465}$

Zembylas, M. (2013). The Emotional Complexities of "Our" and "Their" Loss: The Vicissitudes of Teaching about/for Empathy in a Conflicting Society. Anthropology \& Education Quarterly, 44, 19-37. doi: http://dx.doi.org/10.1111/j.15481492.2012.01175.x

Zhou, Q., Valiente, C. \& Eisenberg, N. (2003). Empathy and its measurement. En Shane J. Lopez y C.R. Snyder (Eds.), Positive psychological assessment: A handbook of models and measures (pp. 269-284). Washington: American Psychological Association.

\section{AGRADECIMIENTOS}

El presente estudio se deriva de algunas conclusiones obtenidas en los proyectos con referencias SEJ2005-00331 y BU030A08, que confirman la importancia de delimitar mejor el constructo de la empatía cuando se mide en contextos de diversidad cultural. Esta nueva concepción está siendo aplicada en un proyecto I+D+I en ejecución, titulado 'El AprendizajeServicio y la Innovación Educativa en la Universidad Española. Un programa centrado en la mejora del rendimiento académico y el capital social de los estudiantes' (Ref. EDU 2013-41687R). Los dos primeros autores del artículo participan en los tres proyectos citados. 
González-González, Hugo; Álvarez-Castillo, José-Luis \& Fernández-Caminero, Gemma (2015). Desarrollo y validación de una escala de medida de la empatía intercultural. RELIEVE, 21 (2), art. 3. DOI: http://dx.doi.org/10.7203/relieve.21.2.7841

\title{
Autores / Authors
}

To know more / Saber más

González-González, Hugo (hugo.gonzalez@uco.es).

Doctor en Ciencias de la Educación y Profesor Ayudante Doctor del Departamento de Educación de la Universidad de Córdoba. Es el autor de contacto para este artículo. Miembro del grupo de investigación "Educación, diversidad y sociedad” del Plan Andaluz de Investigación. Coordinador del Área de Teoría e Historia del Departamento de Educación en la Facultad de Ciencias de la Educación de la UCO. Líneas de investigación: educación inclusiva e intercultural, educación moral, cognición social y estrategias de reducción del prejuicio. Dirección postal: Departamento de Educación. Universidad de Córdoba. Av. San Alerto Magno s/n 14074 - Córdoba.

\begin{abstract}
Álvarez-Castillo, José-Luis (jlalvarez@uco.es).
Doctor en Ciencias de la Educación y Profesor Titular de Universidad del Departamento de Educación de la Universidad de Córdoba. Director del Programa de Doctorado en Educación. Coordinador del grupo de investigación "Educación, diversidad y sociedad" del Plan Andaluz de Investigación. Ha sido Director de Formación de Profesorado e Innovación Docente de la Universidad de Córdoba. Líneas de investigación: educación inclusiva e intercultural, cognición social y estrategias de reducción del prejuicio. Dirección postal: Departamento de Educación. Universidad de Córdoba. Av. San Alerto Magno s/n 14074 - Córdoba.
\end{abstract}

ORCID

0000-0001-7493-4664

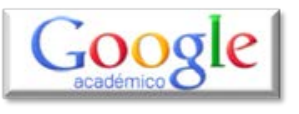

ResearchGate

academia.edu

Fernández-Caminero, Gemma (gemma.fernandez@uco.es).

Máster en Educación inclusiva y Profesora Sustituta Interina del Departamento de Educación de la Universidad de Córdoba. Miembro del grupo de investigación "Educación, diversidad y sociedad" del Plan Andaluz de Investigación. Coordinadora del Grado de Educación Social en la Facultad de Ciencias de la Educación de la UCO. Líneas de investigación: educación inclusiva e intercultural, educación social, educación para la convivencia

\section{RELIEVE}

\section{Revista ELectrónica de Investigación y EValuación Educativa E-Journal of Educational Research, Assessment and Evaluation}

[ISSN: 1134-4032]

(C) Copyright, RELIEVE. Reproduction and distribution of this articles it is authorized if the content is no modified and their origin is indicated (RELIEVE Journal, volume, number and electronic address of the document).

(C) Copyright, RELIEVE. Se autoriza la reproducción y distribución de este artículo siempre que no se modifique el contenido y se indique su origen (RELIEVE, volumen, número y dirección electrónica del documento). 